\title{
Collision-duration time for optical-phonon emission in semiconductors
}

\author{
Paolo Bordone, ${ }^{*}$ Dragica Vasileska, and David K. Ferry \\ Center for Solid State Electronics Research, Arizona State University, Tempe, Arizona 85287-6206
}

(Received 28 April 1995)

\begin{abstract}
The time required to emit an optical (polar and intervalley) phonon by a nearly-free electron in a semiconductor is evaluated using a nonequilibrium Green's-function formalism. The leading idea of the work is that the so-called "collision duration" is related to the time required to build up correlation between the initial and the final state, and then to destroy this correlation as the collision is completed. The use of the nonequilibrium Green's-function formalism gives us the possibility to evaluate explicitly the effects of the correlations in time. Our approach is based on two crucial assumptions: we build the self-energy from only the polarization field of the polar-optical phonon; that is, the self-energy is a function of a single time and position, and we introduce the electron correlation function between the initial and the final states, written in terms of a generalized less-than Green's function in the momentum variables. We derive an analytical expression for the probability for a carrier to end up in a final state $\mathbf{k}$ as a consequence of the emission of a phonon as a function of time. We find that the probability rises to the "Fermi golden rule" result within a few femtoseconds. If the total lifetime broadening of the initial state is comparable to the scattering time, the probability oscillates as it approaches the asymptotic value. For larger initial-state broadening (due to more scattering processes), these oscillations disappear.
\end{abstract}

\section{INTRODUCTION}

In the traditional treatment of the Boltzmann equation for electron transport in semiconductors, collisions are, in general, assumed instantaneous in time and pointlike in space. Since the interactions between the particles and the scattering agents have finite duration, this assumption is not correct in general, not even in classical theory. Thus, scattering normally requires a duration time. The general problem of the time required for a perturbative process to occur has been of interest for many years. Emin ${ }^{1,2}$ studied the lattice relaxation time in a small-polaron hopping motion, which built upon earlier work in this area. ${ }^{3,4}$ Geltman $^{5}$ investigated the time evolution of the ionization probability of a simple onedimensional model atom under the influence of a harmonic electric field as well as a linearly polarized light, by the numerical solution of the time-dependent Schrödinger equation. He found that the duration of the radiation pulse is of crucial importance, as multiphoton absorption was seen to require a certain minimum time to develop, and that processes that are forbidden in the long-time limit by energy conservation are important at short times. Lane ${ }^{7}$ and Mittleman and Tip $^{8}$ have examined the early decay of a prepared state. In particular, Lane, analyzing the decay of a proton in a nucleus, pointed out that such a decay in the pre-Fermi "golden rule" period is usually larger, except at very tiny times. On the other hand, Robinson, facing the problem of the early-time decay rate of a model system by examining the evolution of the probability current at a detector located at macroscopic distance from the source, proposed that such deviations from Fermi's "golden rule" would not be observable, ${ }^{9}$ although this is no longer believed to be the case. Lipavsky et al. ${ }^{10}$ have recently used a nonequilibrium Green's-function method to investigate the time dependence for phonon emission by an electron in semiconductors, but had trouble achieving convergent results. Nevertheless, they find that at equilibrium the noninterference of subsequent processes is given by a single characteristic time scale, and that an estimator of this time scale is given approximately by the Landau liquid theory as $h / E$, where $E$ is measured from the threshold for the process under consideration.

Over the past few years, the development of lasers with pulse lengths as short as 6 fs has made it possible to probe very fast nonequilibrium properties of electron-hole plasmas in semiconductors. ${ }^{11}$ It is apparent that electron-electron scattering provides randomization of the initial distribution of photoexcited carriers on times as fast as a few tens of femtoseconds. ${ }^{12,13}$ Nevertheless, detailed Monte Carlo studies have indicated that a finite collision duration could affect the observed relaxation of the hot electron plasma. ${ }^{14}$

A surprisingly unified picture emerges from the previously mentioned work, although the earlier work dealt with localized electrons and the later work with nearly-free electrons (describable by a single momentum state). All of the studies are concerned with a perturbative regime of single electron-photon (or phonon) events that can be treated by perturbation theory. In all cases, the transition rate approaches a constant value at long times (in three dimensions). A key factor, however, is that the so-called "collision duration" is related to the time required to build up correlation between the initial and final states, and then to destroy this correlation as the collision is completed. Both Geltman $^{5,6}$ and Lipavsky et al. ${ }^{10}$ find that the approach to the asymptotic form for long times shows weak oscillations about the asymptotic result. In Geltman's case, these oscillations rise above unit probability (in units of the Fermi "golden rule" result), indicating that the probability of scattering is actually enhanced for short periods of time just before the constant-value plateau. On the other hand, Lipavsky et al. find that the oscillations cause decreases in the probability for short periods of time just before the plateaus.

Here, our aim is to study the time required to emit an 
optical phonon by a nearly-free electron in semiconductors. Our approach follows the pioneering ideas developed by Kuhn and Rossi ${ }^{15}$ and Haug ${ }^{16,17}$ to study ultrafast processes in photoexcited semiconductors, where details such as the Rabi oscillations are important. The electron is assumed to interact with the (polar or intervalley) optical phonon through the polarization field of the phonon, first building up a correlation between the initial and final states, and then breaking up this correlation as the collision is completed. In other words we can say that the electron is in a well-defined state of the momentum before the scattering event (initial state), and that it will be in a well-defined state of the momentum after the scattering is completed (final state). During the scattering the electron state is not exactly defined. This condition will be mathematically described in terms of a generalized less-than Green's function, that can be seen as a polarization induced between the initial and the final state by the perturbation potential. A particle is destroyed at the time $t_{1}$ in the state $\mathbf{k}_{1}$ and is created at the time $t_{2}$ in the state $\mathbf{k}_{2}$. We define the "collision duration time" as the time over which this generalized less-than Green's function is different from zero, or, alternatively, over which the electron appreciably feels the polarization field, but we will find the actual value to be somewhat arbitrary. We return to this point later. The calculations are developed using the nonequilibrium Green's-function formalism, which gives us the possibility to evaluate explicitly the effect of the correlations in time, and in single scattering event condition. We find, contrary to the earlier results, that the presence of oscillations in the approach to the plateau values depends upon the collisional broadening of the initial state. Oscillations only occur when this broadening is sufficiently narrow, and then appear in a manner consistent with the earlier results of Geltman and Lane, with oscillations above the unit probability amplitude.

The paper is organized as follows. In Sec. II, we present the theoretical model and derive the equation of motion for the less-than Green's function. A first formal integration in time and a consequent Laplace transformation of this equation for the polar-optical phonon case are given in Sec. III. In Sec. IV, we present the analytical derivation of the scattering rate. In Sec. V, we summarize the calculations for the case of the intervalley optical phonon. The results are presented and discussed in Sec. VI.

\section{THEORETICAL MODEL}

Our starting point is the equations of motion for the nonequilibrium Green's function: ${ }^{18-21}$

$$
\begin{aligned}
& \left(i \hbar \frac{\partial}{\partial t_{1}}+\frac{\hbar^{2} \nabla_{1}^{2}}{2 m}\right) \tilde{G}\left(\mathbf{r}_{1}, t_{1} ; \mathbf{r}_{2}, t_{2}\right)=\hbar \tilde{I} \\
& \quad+\iint d^{3} r_{3} d t_{3} \tilde{\Sigma}\left(\mathbf{r}_{1}, t_{1} ; \mathbf{r}_{3}, t_{3}\right) \tilde{G}\left(\mathbf{r}_{3}, t_{3} ; \mathbf{r}_{2}, t_{2}\right) \\
& \left(-i \hbar \frac{\partial}{\partial t_{2}}+\frac{\hbar^{2} \nabla_{2}^{2}}{2 m}\right) \tilde{G}\left(\mathbf{r}_{1}, t_{1} ; \mathbf{r}_{2}, t_{2}\right)=\hbar \tilde{I} \\
& \quad+\iint d^{3} r_{3} d t_{3} \tilde{G}\left(\mathbf{r}_{1}, t_{1} ; \mathbf{r}_{3}, t_{3}\right) \tilde{\Sigma}\left(\mathbf{r}_{3}, t_{3} ; \mathbf{r}_{2}, t_{2}\right),
\end{aligned}
$$

where $\tilde{G}$ and $\tilde{\Sigma}$ are matrices defined as

$$
\tilde{G}=\left(\begin{array}{cc}
G^{t} & G^{<} \\
G^{>} & G^{\bar{t}}
\end{array}\right) \quad \text { and } \tilde{\Sigma}=\left(\begin{array}{cc}
\Sigma^{t} & \Sigma^{<} \\
\Sigma^{>} & \Sigma^{\bar{t}}
\end{array}\right)
$$

and $\tilde{I}$ is the identity matrix. Here the superscripts $t$ and $\bar{t}$ refer to the time-ordered and anti-time-ordered Green's functions $G^{t}=(-i / \hbar)\left\langle T_{c}\left\{\Psi\left(\mathbf{r}_{1}, t_{1}\right) \Psi^{+}\left(\mathbf{r}_{2}, t_{2}\right)\right\}\right\rangle$, where $T_{c}$ is the time-ordering operator along the contour $c$ and the angular brackets refer to an ensemble average over the nonequilibrium electronic states of the system. The general state of the system is described in terms of electron and phonon states. Here we consider negligible the perturbation induced by the electrons on the phonon population, which means that we can factorize the states of the system in terms of products of electrons and equilibrium phonon states. Since the field operators included in the definition of the electron Green's function act only on the electron states, we can eliminate the dependence on the phonon states, summing over them without affecting the form of the Green's function. The selfenergy $\Sigma$ is usually a two-point function, as indicated in the above equations, and is obtained from a diagrammatic expansion of the perturbation interaction. Here, however, we make two crucial deviations from the normal approach. First, we follow the approach of Kuhn-Rossi ${ }^{15}$ and Haug ${ }^{16,17}$ (who work with the electromagnetic field of the photon) by introducing only the polarization field of the longitudinal polar optical phonon

$$
\Sigma(\mathbf{r}, t)=\sum_{\mathbf{q}} \lambda_{q}\left(a_{q}^{\dagger} e^{-i \mathbf{q} \cdot \mathbf{r}} e^{i \omega_{0} t}+a_{q} e^{i \mathbf{q} \cdot \mathbf{r}} e^{-i \omega_{0} t}\right) .
$$

In the case of the longitudinal (polar) optical phonon, we can write the coupling constant as

$$
\lambda_{q}=\frac{1}{q}\left(\frac{\hbar e^{2}}{2 \eta V \omega_{0}}\right)^{1 / 2}, \quad \frac{1}{\eta}=\omega_{0}^{2}\left(\frac{1}{\varepsilon_{\infty}}-\frac{1}{\varepsilon_{0}}\right),
$$

where $\omega_{0}$ is the longitudinal optical phonon frequency, $V$ is the volume, and $\varepsilon_{0}$ and $\varepsilon_{\infty}$ are the static and high-frequency dielectric function, respectively. Alternatively, in the intervalley optical phonon case, the coupling constant becomes

$$
\lambda_{q}=\left(\frac{\hbar D^{2}}{2 \rho V \omega_{0}}\right)^{1 / 2},
$$

where $D$ is the coupling constant for the intervalley process, and $\rho$ is the mass density of the material. Here $a_{q}^{\dagger}$ and $a_{q}$ are the creation and annihilation operators for the phonon mode $\mathbf{q}$, respectively. It should be noted that the present approach is quite general, and should work in principle with other values for $\lambda_{q}$. This choice of the self-energy corresponds to the electron interacting with the dipole field of the phonon and makes the self-energy matrix diagonal, as the self-energy is a single-point function (function of a single time and position). This also leads to a cancellation of the integration in the space and time variables in (1) and (2). Thus, we can separate the equations for the less-than functions from the matrix formulation.

The second crucial assumption, also following KuhnRossi and Haug, is to explicitly introduce the electron correlation function between the initial and final states. For this, we then introduce the generalized less-than Green's function (in Fourier-transformed notation) 


$$
G^{<}=G^{<}\left(\mathbf{k}_{1}, t_{1} ; \mathbf{k}_{2}, t_{2}\right)=i\left\langle\Psi_{\mathbf{k}_{2}}^{\dagger}\left(t_{2}\right) \Psi_{\mathbf{k}_{1}}\left(t_{1}\right)\right\rangle .
$$

When the two momentum arguments are equal, we have the normal less-than Green's function at either the initial momentum $\mathbf{k}_{2}$ or the final $\mathbf{k}_{1}$. The new electron correlation polarization, however, is defined between the states $\mathbf{k}_{2}$ and $\mathbf{k}_{1}$, in a manner analogous to the electron-hole polarization discussed in Refs. 15-17. In a sense, this is an improper Green's function that vanishes in equilibrium and in steady state. We now write the less-than function as a new $2 \times 2$ matrix with the functions for the two momentum states on the diagonal and the electron polarization, and its complex conjugate, on the off-diagonal. The self-energy term is also written as a $2 \times 2$ matrix, with the interaction field in the off-diagonal position, as it connects the less-than Green's function with the polarization.

Now we will develop the details of the calculations for the polar-optical phonons case, while the procedure for the intervalley phonons scattering mechanism will be summarized in Sec. V.

Fourier transforming with respect to $\mathbf{r}_{1}-\mathbf{r}_{2}$, we get, in the polar-optical emission case, the equations of motion for the general Green's function

$$
\begin{gathered}
\left(i \hbar \frac{\partial}{\partial t_{1}}-\frac{\hbar^{2} k_{1}^{2}}{2 m}\right) G^{<}\left(\mathbf{k}_{1}, t_{1} ; \mathbf{k}_{2}, t_{2}\right)=\left(\frac{\hbar e^{2}}{2 \eta \omega_{0}}\right)^{1 / 2} \int \frac{d^{3} q}{(2 \pi)^{3 / 2}} \frac{\left(n_{q}+1\right)^{1 / 2}}{q} e^{i \omega_{0} t_{1}} G^{<}\left(\mathbf{k}_{1}-\mathbf{q}, t_{1} ; \mathbf{k}_{2}, t_{2}\right), \\
\left(-i \hbar \frac{\partial}{\partial t_{2}}-\frac{\hbar^{2} k_{2}^{2}}{2 m}\right) G^{<}\left(\mathbf{k}_{1}, t_{1} ; \mathbf{k}_{2}, t_{2}\right)=\left(\frac{\hbar e^{2}}{2 \eta \omega_{0}}\right)^{1 / 2} \int \frac{d^{3} q}{(2 \pi)^{3 / 2}} \frac{\left(n_{q}+1\right)^{1 / 2}}{q} e^{-i \omega_{0} t_{2}} G^{<}\left(\mathbf{k}_{1}, t_{1} ; \mathbf{k}_{2}+\mathbf{q}, t_{2}\right) .
\end{gathered}
$$

With these ideas in mind, we rewrite these equations for the specific Green's functions of interest

$$
\begin{gathered}
\left(-i \hbar \frac{\partial}{\partial t_{2}}-\varepsilon_{k}\right) G^{<}\left(\mathbf{k}, t_{1}, t_{2}\right)=\left(\frac{\hbar e^{2}}{2 \eta \omega_{0}}\right)^{1 / 2} \int \frac{d^{3} q}{(2 \pi)^{3 / 2}} \frac{\left(n_{q}+1\right)^{1 / 2}}{q} e^{-i \omega_{0} t_{2}} G^{<}\left(\mathbf{k}, t_{1} ; \mathbf{k}+\mathbf{q}, t_{2}\right), \\
\left(i \hbar \frac{\partial}{\partial t_{1}}-\varepsilon_{k}\right) G^{<}\left(\mathbf{k}, t_{1} ; \mathbf{k}+\mathbf{q}, t_{2}\right)=\left(\frac{\hbar e^{2}}{2 \eta \omega_{0}}\right)^{1 / 2} \int \frac{d^{3} q^{\prime}}{(2 \pi)^{3 / 2}} \frac{\left(n_{q^{\prime}}+1\right)^{1 / 2}}{q^{\prime}} e^{i \omega_{0} t_{1}} G^{<}\left(\mathbf{k}+\mathbf{q}^{\prime}, t_{1} ; \mathbf{k}+\mathbf{q}, t_{1}\right),
\end{gathered}
$$

where we have replaced $\mathbf{q}^{\prime}$ by $-\mathbf{q}^{\prime}$ in (9) and $\varepsilon_{k}=(\hbar k)^{2} / 2 m^{*}$. Integrating formally in time (7) and (8) we find $\left[G^{<}\left(\mathbf{k}, t_{1}, t_{2}\right) \equiv G^{<}\left(\mathbf{k}, t_{1} ; \mathbf{k}, t_{2}\right]\right.$

$$
\begin{gathered}
G^{<}\left(\mathbf{k}, t_{1}, t_{2}\right)=\frac{i}{\hbar}\left(\frac{\hbar e^{2}}{2 \eta \omega_{0}}\right)^{1 / 2} \int_{0}^{t_{2}} d t_{2}^{\prime} e^{i \omega_{k}\left(t_{2}-t_{2}^{\prime}\right)} e^{-i \omega_{0} t_{2}^{\prime}} \int \frac{d^{3} q}{(2 \pi)^{3 / 2}} \frac{\left(n_{q}+1\right)^{1 / 2}}{q} G^{<}\left(\mathbf{k}, t_{1} ; \mathbf{k}+\mathbf{q}, t_{2}^{\prime}\right), \\
G^{<}\left(\mathbf{k}, t_{1} ; \mathbf{k}+\mathbf{q}, t_{2}\right)=-\frac{i}{\hbar}\left(\frac{\hbar e^{2}}{2 \eta \omega_{0}}\right)^{1 / 2} \int_{0}^{t_{1}} d t_{1}^{\prime} e^{-i \omega_{k}\left(t_{1}-t_{1}^{\prime}\right)} e^{i \omega_{0} t_{1}^{\prime}} \int \frac{d^{3} q^{\prime}}{(2 \pi)^{3 / 2}} \frac{\left(n_{q^{\prime}}+1\right)^{1 / 2}}{q^{\prime}} G^{<}\left(\mathbf{k}+\mathbf{q}^{\prime}, t_{1}^{\prime} ; \mathbf{k}+\mathbf{q}, t_{2}\right),
\end{gathered}
$$

where $\omega_{k}=\hbar k^{2} /(2 m)$ is the frequency corresponding to the electron wave vector $\mathbf{k}$. We have now achieved, from the equations of motion, an equation for $G^{<}\left(\mathbf{k}, t_{1}, t_{2}\right)$ in terms of the correlation between the states $\mathbf{k}$ and $\mathbf{k}+\mathbf{q}$. We may then eliminate the electron polarization $G^{<}\left(\mathbf{k}, t_{1} ; \mathbf{k}+\mathbf{q}, t_{2}\right)$ substituting (10) into (9), which leads to

$$
\begin{aligned}
G^{<}\left(\mathbf{k}, t_{1}, t_{2}\right)= & \frac{1}{\hbar^{2}}\left(\frac{\hbar e^{2}}{2 \eta \omega_{0}}\right) e^{-i \omega_{k}\left(t_{1}-t_{2}\right)} \int_{0}^{t_{1}} d t_{1}^{\prime} \int_{0}^{t_{2}} d t_{2}^{\prime} e^{i\left(\omega_{k}+\omega_{0}\right)\left(t_{1}^{\prime}-t_{2}^{\prime}\right)} \int \frac{d^{3} q}{(2 \pi)^{3 / 2}} \frac{\left(n_{q}+1\right)^{1 / 2}}{q} \\
& \times \int \frac{d^{3} q^{\prime}}{(2 \pi)^{3 / 2}} \frac{\left(n_{q^{\prime}}+1\right)^{1 / 2}}{q^{\prime}} G^{<}\left(\mathbf{k}+\mathbf{q}^{\prime}, t_{1}^{\prime} ; \mathbf{k}+\mathbf{q}, t_{2}^{\prime}\right) .
\end{aligned}
$$

We now recognize that, from the physical point of view, the only contribution in (11) has to come from the one-particle less-than Green's function, which requires only a single momentum in a homogeneous system. ${ }^{18}$ Hence, we find an equation for $G^{<}\left(\mathbf{k}, t_{1}, t_{2}\right)$ in terms of $G^{<}\left(\mathbf{k}+\mathbf{q}, t_{1}^{\prime}, t_{2}^{\prime}\right)$ :

$$
G^{<}\left(\mathbf{k}, t_{1}, t_{2}\right)=\left(\frac{e^{2}}{2 \hbar \eta \omega_{0}}\right) e^{-i \omega_{k}\left(t_{1}-t_{2}\right)} \int \frac{d^{3} q}{(2 \pi)^{3}} \frac{\left(n_{q}+1\right)}{q^{2}} \int_{0}^{t_{1}} d t_{1}^{\prime} \int_{0}^{t_{2}} d t_{2}^{\prime} e^{i\left(\omega_{k}+\omega_{0}\right)\left(t_{1}^{\prime}-t_{2}^{\prime}\right)} G^{<}\left(\mathbf{k}+\mathbf{q}, t_{1}^{\prime}, t_{2}^{\prime}\right) .
$$

From the physical point of view, when $t_{1}=t_{2}=t,(12)$ is an equation for the occupation number of the state $\mathbf{k}$ at time $t$, that reads

$$
G^{<}(\mathbf{k}, t)=\left(\frac{e^{2}}{2 \hbar \eta \omega_{0}}\right) \int \frac{d^{3} q}{(2 \pi)^{3}} \frac{\left(n_{q}+1\right)}{q^{2}} \int_{0}^{t} d t_{1}^{\prime} \int_{0}^{t} d t_{2}^{\prime} e^{i\left(\omega_{k}+\omega_{0}\right)\left(t_{1}^{\prime}-t_{2}^{\prime}\right)} G^{<}\left(\mathbf{k}+\mathbf{q}, t_{1}^{\prime}, t_{2}^{\prime}\right) .
$$


In order to reduce the two-time propagator on the righthand side (RHS), we introduce the generalized KadanoffBaym ansatz ${ }^{22}$ for the initial state

$$
\begin{aligned}
G^{<}\left(\mathbf{k}+\mathbf{q}, t_{1}^{\prime}, t_{2}^{\prime}\right)= & i\left[G^{r}\left(\mathbf{k}+\mathbf{q}, t_{1}^{\prime}, t_{2}^{\prime}\right) f\left(t_{2}^{\prime}\right)\right. \\
& \left.-f\left(t_{1}^{\prime}\right) G^{a}\left(\mathbf{k}+\mathbf{q}, t_{1}^{\prime}, t_{2}^{\prime}\right)\right] .
\end{aligned}
$$

Here $f(t)$ is the particle distribution function of the initial state $\mathbf{k}+\mathbf{q}$ at time $t$, which can be seen either as the particle density matrix at time $t$ or as the particle less-than Green's function of the state $\mathbf{k}+\mathbf{q}$ in the limit in which the two times in the argument coincide. $G^{r}$ and $G^{a}$ are the retarded and advanced Green's functions, which may be written as

$$
\begin{gathered}
G^{r}\left(\mathbf{k}+\mathbf{q}, t_{1}^{\prime}, t_{2}^{\prime}\right) \approx-i \theta\left(t_{1}^{\prime}-t_{2}^{\prime}\right) e^{\left(-i \omega_{k+q}-\gamma\right)\left(t_{1}^{\prime}-t_{2}^{\prime}\right)}, \\
G^{a}\left(\mathbf{k}+\mathbf{q}, t_{1}^{\prime}, t_{2}^{\prime}\right) \approx i \theta\left(t_{2}^{\prime}-t_{1}^{\prime}\right) e^{\left(i \omega_{k+q}-\gamma\right)\left(t_{2}^{\prime}-t_{1}^{\prime}\right)},
\end{gathered}
$$

where $\gamma$ is the imaginary part of the self-energy of the initial state (which we take to be time independent and which is defined as the lifetime of the initial state) and $\omega_{k+q}$ is the frequency corresponding to the electron wave vector $\mathbf{k}+\mathbf{q}$. Substituting (15) into (14), $G^{<}(\mathbf{k}, t)$ is the occupation number of the state of momentum $\mathbf{k}$ at time $t$, where $\mathbf{k}$ is the final state of a polar-optical-phonon emission, and all the possible initial states are considered. These relations, in contrast to the usual Kadanoff-Baym ansatz, take the causality correctly into account. Depending on the sequence of the time arguments the off-diagonal propagator $G^{<}\left(\mathbf{k}+\mathbf{q}, t_{1}^{\prime}, t_{2}^{\prime}\right)$ develops from its equal-time value in terms of retarded or advanced Green's function. These relations are exact for noninteracting particles. Note that in the equal-time limit the nondiagonal spectral function vanishes, because the equal-time anticommutators vanish for these functions. Therefore, the equaltime limit of the generalized Kadanoff-Baym ansatz remains exact in the matrix extension. ${ }^{17}$ Now, (13) reads

$$
\begin{aligned}
G^{<}(\mathbf{k}, t)= & \left(\frac{e^{2}}{2 \hbar \eta \omega_{0}}\right) \int \frac{d^{3} q}{(2 \pi)^{3}} \frac{\left(n_{q}+1\right)}{q^{2}} \int_{0}^{t} d t_{1}^{\prime} \int_{0}^{t} d t_{2}^{\prime}\left\{\theta\left(t_{1}^{\prime}-t_{2}^{\prime}\right) e^{i\left(\omega_{k}+\omega_{0}-\omega_{k+q}\right) t_{1}^{\prime}} e^{-\gamma t_{1}^{\prime}} e^{-i\left(\omega_{k}+\omega_{0}-\omega_{k+q}\right) t_{2}^{\prime}} e^{\gamma t_{2}^{\prime}} f\left(t_{2}^{\prime}\right)\right. \\
& \left.+\theta\left(t_{2}^{\prime}-t_{1}^{\prime}\right) e^{i\left(\omega_{k}+\omega_{0}-\omega_{k+q}\right) t_{1}^{\prime}} e^{\gamma t_{1}^{\prime}} e^{-i\left(\omega_{k}+\omega_{0}-\omega_{k+q}\right) t_{2}^{\prime}} e^{-\gamma t_{2}^{\prime}} f\left(t_{1}^{\prime}\right)\right\}
\end{aligned}
$$

\section{INTEGRATION IN TIME AND LAPLACE TRANSFORMATION}

We can perform a first integration in time of (16). In fact, the first term on the RHS can be integrated over $t_{1}^{\prime}$ and the same can be done with $t_{2}^{\prime}$ in the second term. Developing the calculations leads to

$$
\begin{aligned}
G^{<}(\mathbf{k}, t)= & 2\left(\frac{e^{2}}{2 \hbar \eta \omega_{0}}\right) \int \frac{d^{3} q}{(2 \pi)^{3}} \frac{\left(n_{q}+1\right)}{q^{2}}\left\{\frac{\gamma}{\gamma^{2}+\alpha^{2}} \int_{0}^{t} d t^{\prime} f\left(t^{\prime}\right)-\int_{0}^{t} d t^{\prime} f\left(t^{\prime}\right) e^{-\gamma\left(t-t^{\prime}\right)\left[\frac{\gamma}{\gamma^{2}+\alpha^{2}} \cos \left[\alpha\left(t-t^{\prime}\right)\right]\right.}\right. \\
& \left.\left.-\frac{\alpha}{\gamma^{2}+\alpha^{2}} \sin \left[\alpha\left(t-t^{\prime}\right)\right]\right]\right\}
\end{aligned}
$$

where $\alpha=\omega_{k}+\omega_{0}-\omega_{k+q} \cdot G^{<}(\mathbf{k}, t)$ is now expressed as the difference of two terms. The first term is proportional to a Lorentzian, which is the collision-broadened Fermi "golden rule" that arises from the initial-state lifetime. The second term is a function of two Lorentzians, one due to the initialstate lifetime and one due to the nonconservation of the energy in the short-time limit. This latter term decays in time and arises from the condition in which the two internal times evolve independently $\left(t_{1}^{\prime} \neq t_{2}^{\prime}\right)$. If we assume that the electron is in state $\mathbf{k}+\mathbf{q}$ at $t=0$, then the time derivative of $G^{<}(\mathbf{k}, t)$ gives the probability for an electron to end up in the state $\mathbf{k}$ at time $t$ as a consequence of the emission of a polar-optical phonon, in which the interaction is initiated at $t=0$. Since $\alpha$ depends on the wave vector, the integrations are not straightforward. To verify the behavior of $G^{<}$in the long- and shorttime limits, and get the idea of what to expect as a result, before we perform the actual detailed calculations we Laplace transform (17)

$$
\begin{aligned}
G^{<}(\mathbf{k}, S)= & 2\left(\frac{e^{2}}{2 \hbar \eta \omega_{0}}\right) \int \frac{d^{3} q}{(2 \pi)^{3}} \frac{\left(n_{q}+1\right)}{q^{2}} \frac{1}{\gamma^{2}+\alpha^{2}} \\
& \times\left(\frac{\gamma}{S}-\frac{\gamma^{2}+\gamma S-\alpha^{2}}{(\gamma+S)^{2}+\alpha^{2}}\right) f(S) .
\end{aligned}
$$

According to the Laplace transformation theory, we can now verify the asymptotic behavior of $G^{<}$when $t$ approaches infinity and zero, where the limits are given by the initialand final-value theorem $^{23}$ as $\lim _{t \rightarrow \infty} G^{<}(\mathbf{k}, t)$ $=\lim _{S \rightarrow 0} S G^{<}(\mathbf{k}, S)$ and $\lim _{t \rightarrow 0} G^{<}(\mathbf{k}, t)=\lim _{S \rightarrow \infty} S G^{<}(\mathbf{k}, S)$. In the long-time limit, we find

$$
\lim _{t \rightarrow \infty} G^{<}(\mathbf{k}, t)=2\left(\frac{e^{2}}{2 \hbar \eta \omega_{0}}\right) \int \frac{d^{3} q}{(2 \pi)^{3}} \frac{\left(n_{q}+1\right)}{q^{2}} \frac{\gamma}{\gamma^{2}+\alpha^{2}} f(0)
$$


which is the collision-broadened Fermi "golden rule" multiplied by the initial-state distribution function at zero frequency. Analogously, in the $t=0$ limit

$$
\begin{aligned}
\lim _{t \rightarrow 0} G^{<}(\mathbf{k}, t)= & 2\left(\frac{e^{2}}{2 \hbar \eta \omega_{0}}\right) \int \frac{d^{3} q}{(2 \pi)^{3}} \frac{\left(n_{q}+1\right)}{q^{2}} \frac{1}{\gamma^{2}+\alpha^{2}} \\
& \times(\gamma-\gamma) f(\infty)=0 .
\end{aligned}
$$

Here $G^{<}$is written as the difference of two terms that cancel each other in the very short time range. The time variation then describes the building up of the Fermi "golden rule" result, which is the outcome that is the aim of this paper.

\section{EVALUATION OF THE SCATTERING RATE}

As specified above, the time derivative of $G^{<}(\mathbf{k}, t)$ gives the probability for an electron to wind up in the state $\mathbf{k}$ at time $t$ as a consequence of the emission of a polar optical phonon, in the case for which the interaction begins at $t=0$. The differentiation of (17) gives us

$$
P(\mathbf{k}, t)=\frac{\partial G^{<}}{\partial t}=\frac{2}{(2 \pi)^{2}}\left(\frac{e^{2}}{2 \hbar \eta \omega_{0}}\right) \int d^{3} \mathbf{q} \frac{N_{q}+1}{q^{2}} \int_{0}^{t} d x f(t-x) e^{-\gamma x} \cos (\alpha x) .
$$

To proceed in developing the calculation, we consider $\gamma$ and $N_{q}$ to be independent of $\mathbf{q}$, and constant in time ( $\gamma$ often is dependent upon the energy, but this is weak and has already been ignored by assuming $\gamma$ to be time independent). We also assume that the interaction is weak so that $f(t)$ is also independent of time other than through the overall decay introduced by $\gamma$. Then, we may evaluate (21) as

$$
\begin{aligned}
P(\mathbf{k}, t) & =\frac{2\left(N_{q}+1\right)}{(2 \pi)^{2}}\left(\frac{e^{2}}{2 \hbar \eta \omega_{0}}\right) f(t) \operatorname{Re}\left\{\int \frac{d^{3} \mathbf{q}}{q^{2}} \int_{0}^{t} d x e^{-\gamma x+i \alpha x}\right\} \\
& =\frac{2\left(N_{q}+1\right)}{(2 \pi)^{2}}\left(\frac{e^{2}}{2 \hbar \eta \omega_{0}}\right) f(t) \operatorname{Re}\left\{\int \frac{d^{3} \mathbf{q}}{q^{2}} \frac{1}{\gamma-i \alpha}\left(1-e^{-\gamma x+i \alpha x}\right)\right\} \\
& =\frac{2\left(N_{q}+1\right)}{(2 \pi)^{2}}\left(\frac{e^{2}}{2 \hbar \eta \omega_{0}}\right) f(t) \operatorname{Im}\{\chi(0)-\chi(t)\},
\end{aligned}
$$

where

$$
\chi(0)=-\int \frac{d^{3} \mathbf{q}}{q^{2}} \frac{1}{\alpha+i \gamma}
$$

and

$$
\chi(t)=-\int \frac{d^{3} \mathbf{q}}{q^{2}} \frac{e^{(-\gamma+i \alpha) t}}{\alpha+i \gamma}=\int \frac{d^{3} \mathbf{q}}{|\mathbf{q}-\mathbf{k}|^{2}} \frac{e^{(-\gamma+i \beta) t}}{\beta+i \gamma},
$$

where

$$
\beta=\frac{\hbar k^{2}}{2 m^{*}}-\frac{\hbar q^{2}}{2 m^{*}}+\omega_{0}
$$

The first term can be found directly, following Lewis, ${ }^{24}$ as

$$
I=\operatorname{Im}\{\chi(0)\}=\int \frac{d^{3} \mathbf{q}}{q^{2}} \frac{\gamma}{\alpha^{2}+\gamma^{2}} .
$$

Using the definition of $\alpha$ given below (17), and setting $\mathbf{k}^{\prime}=\mathbf{k}-\mathbf{q}$ and $k_{1}^{2}=k^{2}+2 m^{*} \omega_{0} / \hbar$, we get

$$
I=\gamma\left(\frac{2 m^{*}}{\hbar}\right)^{2} \int \frac{d^{3} \mathbf{q}}{q^{2}} \frac{1}{\left[(q-k)^{2}-k_{1}^{2}-i 2 m^{*} \gamma / \hbar\right]\left[(q-k)^{2}-k_{1}^{2}+i 2 m^{*} \gamma / \hbar\right]},
$$

which is a special case of the integral solved by Lewis. ${ }^{24}$ Using his results, the final result from (27) can be found to be

$$
I=\frac{1}{2}\left(\frac{2 m^{*}}{\hbar \omega_{k}}\right)^{1 / 2} \ln \left[\frac{\left(\sqrt{\omega_{k}+\omega_{0}+i \gamma}+\sqrt{\omega_{k}}\right)}{\left(\sqrt{\omega_{k}+\omega_{0}+i \gamma}-\sqrt{\omega_{k}}\right)} \frac{\left(\sqrt{\omega_{k}+\omega_{0}-i \gamma}+\sqrt{\omega_{k}}\right)}{\left(\sqrt{\omega_{k}+\omega_{0}-i \gamma}-\sqrt{\omega_{k}}\right)}\right] .
$$

The calculation for $\chi(t)$ is more complex, and the details are straightforward. The result, however, may be written as 


$$
\operatorname{Im}\{\chi(t)\}=\pi\left(\frac{\hbar}{2 m^{*} \omega_{k}}\right)^{1 / 2} e^{-\gamma t}\left\{\int_{\omega_{k}+\omega_{0}}^{\infty} d \alpha\left[\frac{\gamma}{\gamma^{2}+\alpha^{2}} \sin (\alpha t)+\frac{a}{\gamma^{2}+\alpha^{2}} \cos (\alpha t)\right] \operatorname{arccot}\left(\frac{\left.\sqrt{\alpha-\left(\omega_{k}+\omega_{0}\right)}\right)}{\sqrt{\omega_{k}}}\right)\right\} .
$$

Finally, substituting the last two equations into (22), the final expression for the probability of scattering into the final state by polar optical phonon emission is found to be

$$
\begin{aligned}
P(\mathbf{k}, t)= & \frac{C}{\sqrt{\omega_{k}}}\left\{\frac{\pi}{4} \ln \left[\frac{\left(\sqrt{\omega_{k}+\omega_{0}+i \gamma}+\sqrt{\omega_{k}}\right)}{\left(\sqrt{\omega_{k}+\omega_{0}+i \gamma}-\sqrt{\omega_{k}}\right)} \frac{\left(\sqrt{\omega_{k}+\omega_{0}-i \gamma}+\sqrt{\omega_{k}}\right)}{\left(\sqrt{\omega_{k}+\omega_{0}-i \gamma}-\sqrt{\omega_{k}}\right)}\right]-e^{-\gamma t} \int_{\omega_{k}+\omega_{0}}^{\infty} d \alpha\left[\frac{\gamma}{\gamma^{2}+\alpha^{2}} \sin (\alpha t)\right.\right. \\
& \left.\left.+\frac{\alpha}{\gamma^{2}+\alpha^{2}} \cos (\alpha t)\right] \operatorname{arccot}\left(\frac{\sqrt{\alpha-\left(\omega_{k}+\omega_{0}\right)}}{\sqrt{\omega_{k}}}\right)\right\},
\end{aligned}
$$

where

$$
C=\frac{1}{2 \pi^{2}}\left(\frac{e^{2}}{\hbar \eta \omega_{0}}\right) \sqrt{\frac{2 m^{*}}{\hbar}} f\left(N_{q}+1\right)
$$

The first term on the RHS represents the Fermi "golden rule." The second term represents the time-dependent buildup of the scattering process. When $t=0$, the integral over the possible initial frequencies of the Lorentzian in $\alpha$ cancels the constant contribution of the Fermi "golden rule." From the physical point of view, we can say that at $t=0$ the scattering is inhibited by the fact that the electron cannot identify a single well-defined phonon energy for the scattering. ${ }^{14}$ Comparing the definition of $\alpha$ below (17) with the equivalent in (30), we see that the integration is now over all possible frequencies of the initial state, at least as these are set by the phonon energy. The individual terms of the integral are the temporal contributions from different energies, each weighted by the Lorentzian reduction for being non-energy-conserving.

\section{INTERVALLEY OPTICAL PHONONS}

In the previous sections we gave the details of the calculations for the polar-optical phonon emission, here we analyze the intervalley optical phonons process. The idea and the method are the same; the only difference is the analytical expression of the self-energy.

The equations of motion for the general Green's function in the emission case read

$$
\begin{gathered}
\left(i \hbar \frac{\partial}{\partial t_{1}}-\frac{\hbar^{2} k_{1}^{2}}{2 m}\right) G^{<}\left(\mathbf{k}_{1}, t_{1} ; \mathbf{k}_{2}, t_{2}\right)=\left(\frac{\hbar D^{2}}{2 \rho \omega_{0}}\right)^{1 / 2} \int \frac{d^{3} q}{(2 \pi)^{3 / 2}}\left(n_{q}+1\right)^{1 / 2} e^{i \omega_{0} t_{1}} G^{<}\left(\mathbf{k}_{1}-\mathbf{q}, t_{1} ; \mathbf{k}_{2}, t_{2}\right) \\
\left(-i \hbar \frac{\partial}{\partial t_{2}}-\frac{\hbar^{2} k_{2}^{2}}{2 m}\right) G^{<}\left(\mathbf{k}_{1}, t_{1} ; \mathbf{k}_{2}, t_{2}\right)=\left(\frac{\hbar D^{2}}{2 \rho \omega_{0}}\right)^{1 / 2} \int \frac{d^{3} q}{(2 \pi)^{3 / 2}}\left(n_{q}+1\right)^{1 / 2} e^{-i \omega_{0} t_{2}} G^{<}\left(\mathbf{k}_{1}, t_{1} ; \mathbf{k}_{2}+\mathbf{q}, t_{2}\right) .
\end{gathered}
$$

Following the same procedure previously used for the polar-optical scattering we find for the occupation number of the state $\mathbf{k}$ at time $t$

$$
\begin{aligned}
G^{<}(\mathbf{k}, t)= & \left(\frac{D^{2}}{2 \hbar \rho \omega_{0}}\right) \int \frac{d^{3} q}{(2 \pi)^{3}}\left(n_{q}+1\right) \int_{0}^{t} d t_{1}^{\prime} \int_{0}^{t} d t_{2}^{\prime}\left\{\theta\left(t_{1}^{\prime}-t_{2}^{\prime}\right) e^{i\left(\omega_{k}+\omega_{0}-\omega_{k+q}\right) t_{1}^{\prime}} e^{-\gamma t_{1}^{\prime}} e^{-i\left(\omega_{k}+\omega_{0}-\omega_{k+q}\right) t_{2}^{\prime}} e^{\gamma t_{2}^{\prime}} f\left(t_{2}^{\prime}\right)\right. \\
& \left.+\theta\left(t_{2}^{\prime}-t_{1}^{\prime}\right) e^{i\left(\omega_{k}+\omega_{0}-\omega_{k+q}\right) t_{1}^{\prime}} e^{\gamma t_{1}^{\prime}} e^{-i\left(\omega_{k}+\omega_{0}-\omega_{k+q}\right) t_{2}^{\prime}} e^{-\gamma t_{2}^{\prime}} f\left(t_{1}^{\prime}\right)\right\} .
\end{aligned}
$$

To display the behavior in time of the occupation number of the state $\mathbf{k}$ we have to carry out the integrations over the time and the phonon wave vector included in (34).

As already done previously for the polar-optical phonons case, we can perform a first integration in time of (33). The first term on the RHS can be integrated over $t_{1}^{\prime}$ and the same can be done with $t_{2}^{\prime}$ in the second term. Developing the calculations leads to

$$
\begin{aligned}
G^{<}(\mathbf{k}, t)= & 2\left(\frac{D^{2}}{2 \hbar \rho \omega_{0}}\right) \int \frac{d^{3} q}{(2 \pi)^{3}}\left(n_{q}+1\right)\left\{\frac{\gamma}{\gamma^{2}+\alpha^{2}} \int_{0}^{t} d t^{\prime} f\left(t^{\prime}\right)-\int_{0}^{t} d t^{\prime} f\left(t^{\prime}\right) e^{-\gamma\left(t-t^{\prime}\right)}\left[\frac{\gamma}{\gamma^{2}+\alpha^{2}} \cos \left[\alpha\left(t-t^{\prime}\right)\right]\right.\right. \\
& \left.\left.-\frac{\alpha}{\gamma^{2}+\alpha^{2}} \sin \left[\alpha\left(t-t^{\prime}\right)\right]\right]\right\}
\end{aligned}
$$


where $\alpha=\omega_{k}+\omega_{0}-\omega_{k+q}$. As in the polar-optical phonon case, $G^{<}(\mathbf{k}, t)$ is now expressed as the difference of two terms. From the comparison between the two expressions, it is clear that the same general considerations valid for (17) apply to (34) as well.

Again, we first examine the short- and long-time behavior by examining the Laplace transform of the above expression. This leads to

$$
\begin{aligned}
G^{<}(\mathbf{k}, S)= & 2\left(\frac{D^{2}}{2 \hbar \rho \omega_{0}}\right) \int \frac{d^{3} q}{(2 \pi)^{3}}\left(n_{q}+1\right) \frac{1}{\gamma^{2}+\alpha^{2}} \\
& \times\left(\frac{\gamma}{S}-\frac{\gamma^{2}+\gamma S-\alpha^{2}}{(\gamma+S)^{2}+\alpha^{2}}\right) f(S) .
\end{aligned}
$$

Even in this case, according to the Laplace transformation theory, we can now verify the asymptotic behavior of $G^{<}$ when $t$ approaches infinity and zero. In the long-time limit, we find

$$
\lim _{t \rightarrow \infty} G^{<}(\mathbf{k}, t)=2\left(\frac{D^{2}}{2 \hbar \rho \omega_{0}}\right) \int \frac{d^{3} q}{(2 \pi)^{3}}\left(n_{q}+1\right) \frac{\gamma}{\gamma^{2}+\alpha^{2}} f(0),
$$

which is the collision-broadened Fermi "golden rule" multiplied by the initial-state distribution function at zero frequency. Analogously, in the $t=0$ limit

$$
\begin{aligned}
\lim _{t \rightarrow 0} G^{<}(\mathbf{k}, t)= & 2\left(\frac{D^{2}}{2 \hbar \rho \omega_{0}}\right) \int \frac{d^{3} q}{(2 \pi)^{3}}\left(n_{q}+1\right) \frac{1}{\gamma^{2}+\alpha^{2}} \\
& \times(\gamma-\gamma) f(\infty) \\
= & 0 .
\end{aligned}
$$

Once again $G^{<}$is written as the difference of two terms that cancel each other in the very short time range.

Using for $\gamma, n_{q}$, and $f(t)$ the same approximations that were applied for the polar-optical phonons process, we can evaluate the scattering rate from the time derivative of (34), as

$$
P(\mathbf{k}, t)=C_{1}\left\{\frac{\pi}{2}\left(\sqrt{\omega_{k}+\omega_{0}+i \gamma}+\sqrt{\omega_{k}+\omega_{0}-i \gamma}\right)-e^{-\gamma t} \int_{\omega_{k}+\omega_{0}}^{\infty} d \alpha\left[\frac{\gamma}{\gamma^{2}+\alpha^{2}} \sin (\alpha t)+\frac{\alpha}{\gamma^{2}+\alpha^{2}} \cos (\alpha t)\right] \sqrt{\alpha-\left(\omega_{k}+\omega_{0}\right)}\right\},
$$

where

$$
C_{1}=\frac{1}{4 \pi^{2}}\left(\frac{D^{2}}{\hbar \rho \omega_{0}}\right)\left(\frac{2 m^{*}}{\hbar}\right)^{3 / 2} f\left(N_{q}+1\right)
$$

and the details of this derivation are also straightforward.

This result is formally similar to (30). The first term on the RHS represents the Fermi "golden rule" while the second represents the time-dependent buildup of the scattering process. The integral in (39) is weakly divergent for $t=0$, but

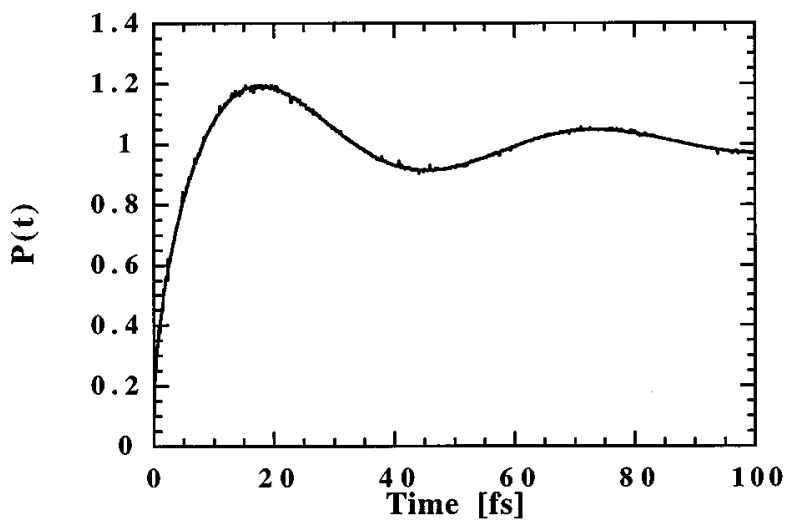

FIG. 1. Calculated scattering rate (normalized to the Fermi "golden rule" value) as a function of time for polar-optical phonon emission in GaAs. The final-state frequency is $\omega_{k}=5.5 \times 10^{13} \mathrm{~s}^{-1}$ and the initial-state broadening is $\gamma=7 \times 10^{12} \mathrm{~s}^{-1}$. this presents no significant difficulty to the evaluation, particularly in relation to the asymptotic forms found above.

\section{RESULTS AND DISCUSSION}

\section{A. Polar-optical phonons}

The integral in (30) is not amenable to an analytical solution, so we evaluate it numerically. In doing this, we consider a bulk GaAs system. The decay parameter $\gamma$ is the imaginary part of the self-energy, and is defined as the lifetime of the initial state. We will use for this a constant value. In fact, for the longitudinal-optical-phonon (LO-phonon) emission, it can be shown ${ }^{25,26}$ that $\gamma$ vanishes below the emission threshold and remains approximately constant above that threshold over a wide range of energies. In Fig. 1, the scattering rate from (30) (normalized to the Fermi "golden rule") is plotted as a function of time for a value of the initial state decay of $\gamma=7 \times 10^{12} \mathrm{~s}^{-1},{ }^{27}$ and for $\omega_{0}=5.5 \times 10^{13} \mathrm{~s}^{-1}$ and $\omega_{k}=\omega_{0}$. These values correspond to the $\mathrm{LO}$ mode parameters and the value of $\gamma$ is taken by an assumption that the initial state decay is dominated by the single interaction. We compare the early-time results in Fig. 2 with the case for a larger value of $\gamma=2.6 \times 10^{14} \mathrm{~s}^{-1}$. As already stated, $\gamma$ is the initial-state lifetime. This means that, in principle, within $\gamma$ are included the effects of all the scattering processes considered in the system. Therefore, increasing $\gamma$ from the value assumed for the LO-phonon scattering means adding other scattering mechanisms to the system. In particular, values of $\gamma$ ranging between $10^{14}$ and $10^{15} \mathrm{~s}^{-1}$ correspond to a physical situation in which the initial-state decay is dominated by rapid scattering, 


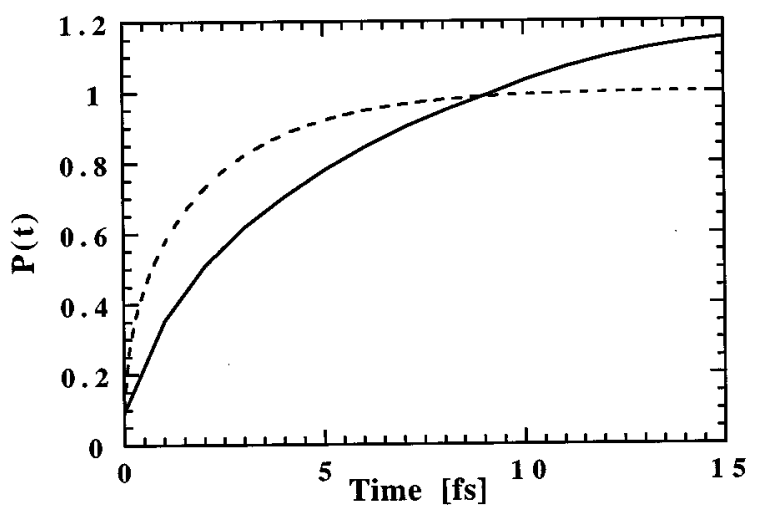

FIG. 2. Polar-optical phonon emission: comparison between the case for $\gamma=7 \times 10^{12} \mathrm{~s}^{-1}$ (solid line) and $\gamma=2.6 \times 10^{14} \mathrm{~s}^{-1}$ (dashed line). These are discussed in the text.

such as arises from the intervalley processes. The scattering rate, in the slow decay case, starts from zero and has a fast rise in the first $10 \mathrm{fs}$, then converges to the Fermi "golden rule" term in the long-time limit with damped oscillations. The rise time is much faster than $e^{-\gamma t}$ because of the fundamental role played by the integral over the frequency that multiplies the exponential factor. The presence of oscillations rising above the Fermi term indicates that the scattering probability is actually enhanced for short periods of time just before the constant-value plateau. This is consistent with the results obtained by Geltman ${ }^{5,6}$ investigating the time required for the ionization of an atom excited by electromagnetic radiation, and by Lane ${ }^{7}$ studying the early decay of a prepared state. For a large value of $\gamma$, however, the probability rises rapidly, and approaches the Fermi "golden rule" value without any oscillations. For values of $\gamma$ ranging between $10^{12}$ and $10^{14} \mathrm{~s}^{-1}$, the time variation of the integral term gives the main contribution to the collision-duration time. As can be seen in Fig. 2, an increase in the value of $\gamma$ produces two main consequences: (1) the collision-duration time decreases, and (2) the oscillations are canceled, leading to a more conventional exponential behavior. The first effect can be understood in terms of energy broadening. As already discussed, on the very short time range, the scattering is inhibited by the impossibility of the electron to identify an appropriate energy. Increasing the initial-state broadening corresponds to increasing the probability for the carrier to access an appropriate energy for the interaction, thus quickening the scattering process. The second effect simply displays the fact that the exponential function has become strong enough to damp any possible long-time oscillations. In Fig. 3 the scattering rates, as a function of time, for three different values of the final-state frequencies are compared. As can be seen, the rise time and the period and the amplitude of the oscillations decrease with increasing frequency. We interpret this behavior in terms of the normal $\sin (x) / x$ behavior that accompanies the Fermi "golden rule". In the latter, higher energies are coupled to shorter times for the same oscillator phase ( $x$ here would be $\omega t$ ). It is this trend that also leads to the Landau liquid behavior.

From Fig. 1, we can define the collision duration time as that time in which the initial and final states are correlated. On the other hand, to assign a precise numerical value to this

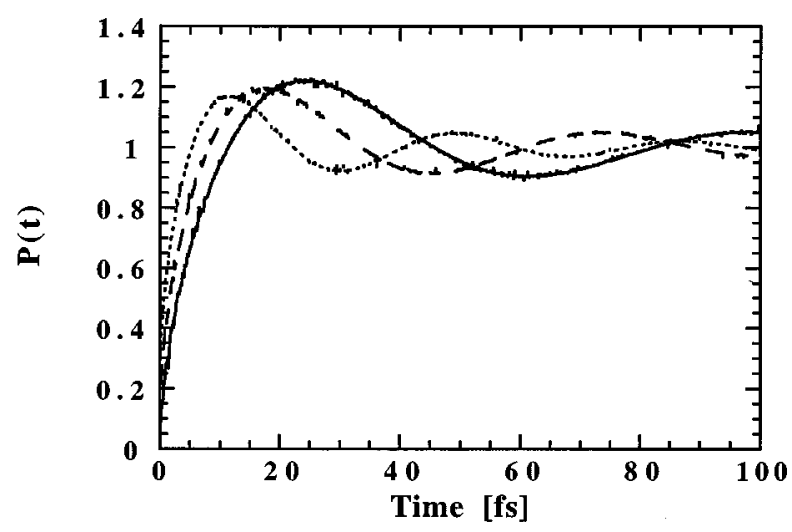

FIG. 3. Polar-optical phonon emission: scattering rates comparison for three different values of the final-state frequency for $\gamma=7 \times 10^{12} \mathrm{~s}^{-1} . \omega_{k}=0.5 \omega_{0}$ (solid line), $\omega_{k}=\omega_{0}$ (dashed line), and $\omega_{k}=2 \omega_{0}$ (dotted line).

time is still arbitrary. The particular analytical form of the scattering rate will be our guide in this choice. In (30) the scattering probability is written as the difference of two terms, the first constant in time, the second given by the product of an exponential decaying factor, due to the initialstate finite lifetime, with an integral over the initial-state frequency. This suggests that one estimate of the collision duration time is the time at which the probability reaches $(1-1 /$ $e) \approx 0.63$ of the asymptotic value. In this way, the contributions of the integral and the exponential factor are comparable. In Fig. 4, the collision duration time, determined from the decay of the initial-state correlation, is plotted as a function of the frequency of the final state. The dashed curve represents the $h / E=2 \pi / \omega_{k}$ behavior expected from the Fermi liquid theory. ${ }^{10}$ Although our data seem to reproduce the Fermi liquid trend very well in the medium frequency region, we find that the collision duration time diverges much slower as the frequency approaches zero. Moreover, the amplitude is clearly different, but the degree by which they differ is sensitive to the definition of the collision-duration time adopted from, e.g., Fig. 1.

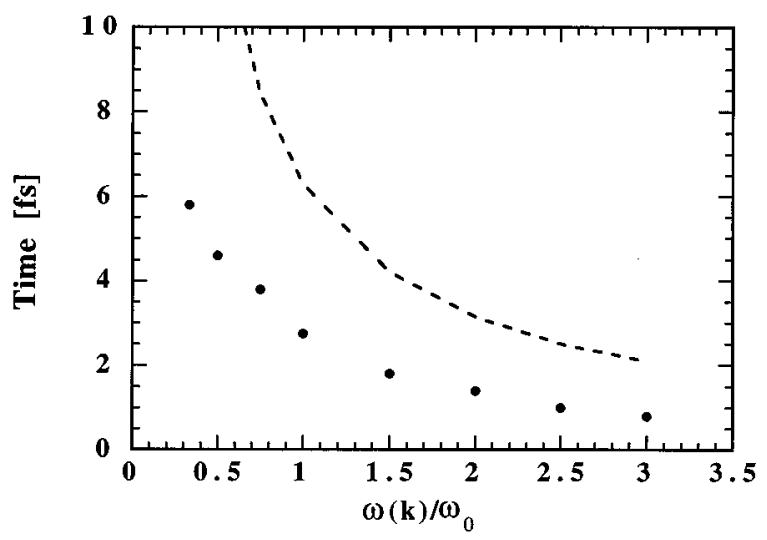

FIG. 4. Polar-optical phonon emission: collison-duration time as a function of the final-state frequency, normalized to the polaroptical-phonon frequency. The dashed curve represents the $2 \pi / \omega_{k}$ behavior expected from Fermi-liquid theory. ${ }^{10}$ 


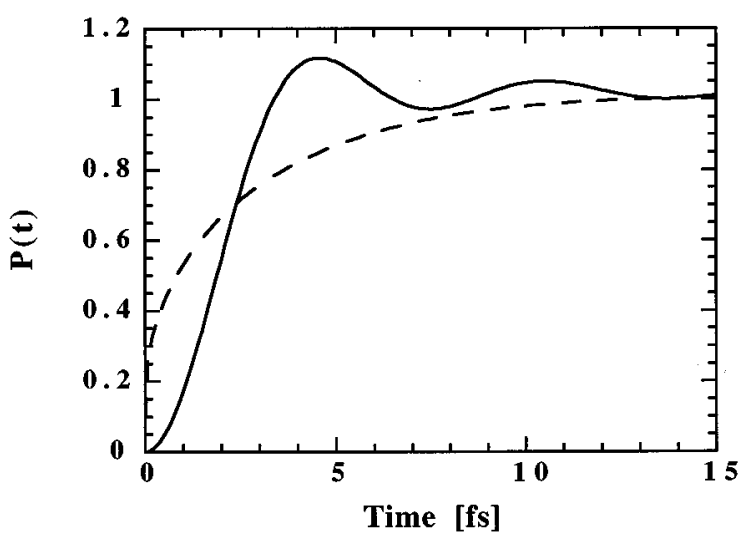

FIG. 5. Calculated scattering rate (normalized to the Fermi "golden rule" value) as a function of time in GaAs. Intervalley optical phonon emission (solid line) for a final-state frequency $\omega_{k}=\omega_{0}=4.7 \times 10^{13} \mathrm{~s}^{-1}$ and an initial state broadening $\gamma=10^{14} \mathrm{~s}^{-1}$ compared with the polar-optical phonon result (dashed line) for $\omega_{k}=\omega_{0}=5.5 \times 10^{13} \mathrm{~s}^{-1}$ and $\gamma=10^{14} \mathrm{~s}^{-1}$.

\section{B. Intervalley phonons}

Once we have determined the frequency cutoff we evaluate the integral in (38) numerically. We consider the same bulk GaAs system used above for the polar-optical mechanism. In the case of the intervalley optical phonon, $\gamma$ is slowly increasing with energy over a wide range of energies $^{26}$ and can be considered with a good approximation constant. In Fig. 5, the solid line represents the scattering rate from (38) (normalized to the Fermi "golden rule") plotted as a function of time for a value of the initial-state decay of $\gamma=10^{14} \mathrm{~s}^{-1},{ }^{20}$ for $\omega_{0}=4.7 \times 10^{13} \mathrm{~s}^{-1}$ (corresponding to the $\Gamma-X$ intervalley optical phonon) and $\omega_{k}=\omega_{0}$. The dashed curve is the result obtained in the polar-optical phonon case, with the same value of $\gamma$, for $\omega_{0}=5.5 \times 10^{13} \mathrm{~s}^{-1}$ and $\omega_{k}=\omega_{0}$. $\gamma$ is chosen by the assumption that the initial-state decay is dominated by the single interaction. The scattering rate, starting from zero, has a fast rise in the first few femtoseconds, then converges to the Fermi "golden rule" term in the longtime limit with damped oscillations. This is the same behavior observed in the polar-optical phonon case, when the much lower values of $\gamma$ (of the order of $10^{12} \mathrm{~s}^{-1}$ ) characteristic of the polar-optical process are used. Again, the rise time is much faster than the one related to $e^{-\gamma t}$ because of the fundamental role played by the integral over the frequency that multiplies the exponential factor. This carries the information of the correlation in time between the initial and the final states of the carrier. For $\delta=10^{14}$ the optical-phonon scattering rate does not show oscillations anymore, though the rise times of the two mechanisms are similar. The difference in the slope of the two curves in the zero time limit displays the different dependence of the self-energies from the phonon wave vector. In Fig. 6 the scattering rates, as a function of time, for three different values of the final-state frequencies are compared. As can be seen, the rise time and the period of the oscillations decrease with increasing frequency. Once again this is the same behavior found in the optical-phonon case.

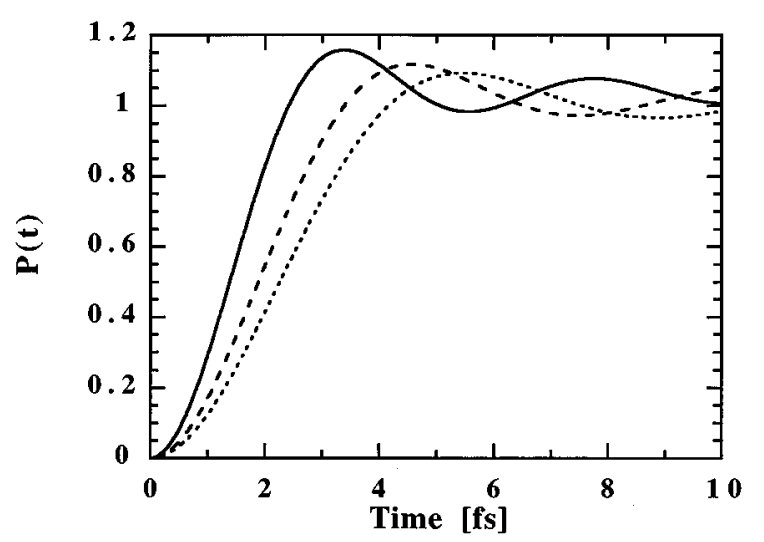

FIG. 6. Intervalley optical phonon emission: scattering rates comparison for three different values of the final-state frequency for $\gamma=10^{14} \mathrm{~s}^{-1} \cdot \omega_{k}=0.5 \omega_{0}$ (dotted line), $\omega_{k}=\omega_{0}$ (dashed line), and $\omega_{k}=2 \omega_{0}$ (solid line).

In Fig. 7, the collision duration time, determined from the decay of the initial-state correlation applying the same criterion used in the polar-optical phonon case, is plotted as a function of the frequency of the final state for $\gamma=1.5 \times 10^{14}$ $\mathrm{s}^{-1}$ (triangles), $\gamma=10^{14} \mathrm{~s}^{-1}$ (diamonds) and $\gamma=0.6 \times 10^{14} \mathrm{~s}^{-1}$ (circles). The collision duration time increases with decreasing $\gamma$. This is the same effect observed in the polar-optical phonon case and can therefore be interpreted in the same way. For this reason the values of the collision duration times for the intervalley scattering mechanism are found to be lower then the ones evaluated for the polar-optical interaction, even if in the latter case the phonon energy is higher. This is shown in Fig. 8, where the curve for $\gamma=10^{14} \mathrm{~s}^{-1}$ and $\omega_{0}=4.7 \times 10^{13} \mathrm{~s}^{-1}$ (diamonds) from Fig. 7 is compared with the result obtained for the polar-optical phonon for $\gamma=7 \times 10^{12} \mathrm{~s}^{-1}$ and $\omega_{0}=4.7 \times 10^{13} \mathrm{~s}^{-1}$ (circles).

In Fig. 9 is presented the comparison between the scattering rate as a function of time for $\gamma=0.6 \times 10^{14} \mathrm{~s}^{-1}$ (solid line) and $\gamma=1.5 \times 10^{14} \mathrm{~s}^{-1}$ (dashed line) for $\omega_{k}=\omega_{0}=4.7 \times 10^{13}$ $\mathrm{s}^{-1}$. Besides the already described effect of the reduction of the collision duration, the increasing of $\gamma$ implies, as in the polar-optical case, the decreasing of the oscillations.

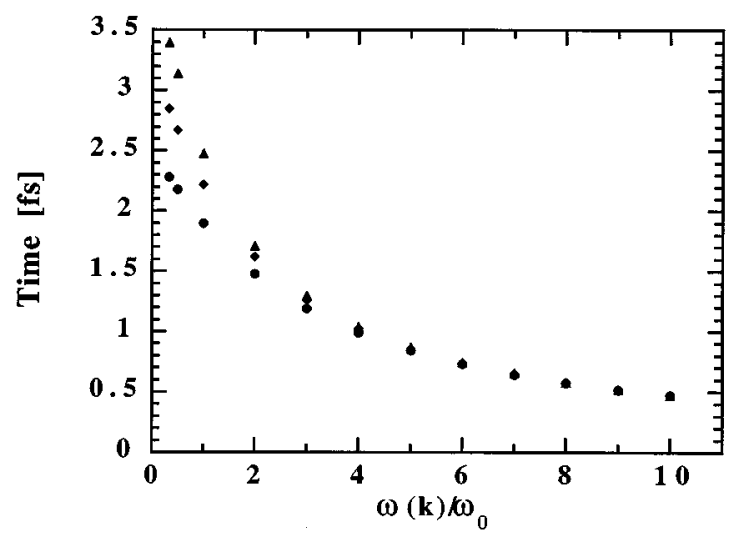

FIG. 7. Intervalley optical phonon emission: collison-duration time as a function of the final-state frequency, normalized to the intervalley optical phonon frequency. $\gamma=1.5 \times 10^{14} \mathrm{~s}^{-1}$ (circles), $\gamma=10^{14} \mathrm{~s}^{-1}$ (diamonds), and $\gamma=0.6 \times 10^{14} \mathrm{~s}^{-1}$ (triangles). 


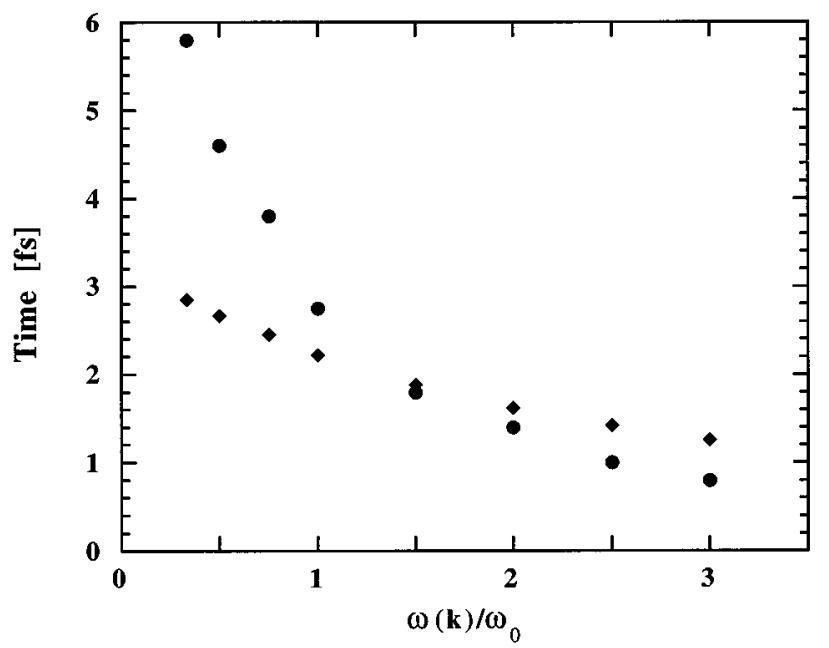

FIG. 8. Collison-duration time as a function of the final-state frequency, normalized to the optical-phonon frequency. Comparison between the result for the intervalley optical phonon with $\gamma=10^{14}$ $\mathrm{s}^{-1}$ and $\omega_{0}=4.7 \times 10^{13} \mathrm{~s}^{-1}$ (diamonds) and the polar-optical phonon with $\gamma=7 \times 10^{12} \mathrm{~s}^{-1}$ and $\omega_{0}=5.5 \times 10^{13} \mathrm{~s}^{-1}$ (circles).

\section{CONCLUSIONS}

We have derived an analytical expression for the probability for a carrier to end up in a final state $\omega_{k}$ as a consequence of the emission of a polar-optical or of an intervally optical phonon as a function of time. For both scattering mechanisms, the probability rises rapidly to the Fermi "golden rule" result, and oscillations in the probability are damped by the decay of the initial state. Our calculations show that the collision duration time, which is found to be of the order of $(1-10) \times 10^{-15} \mathrm{~s}$, can be defined as the time over which the correlation of the initial and final states, induced by the pho-

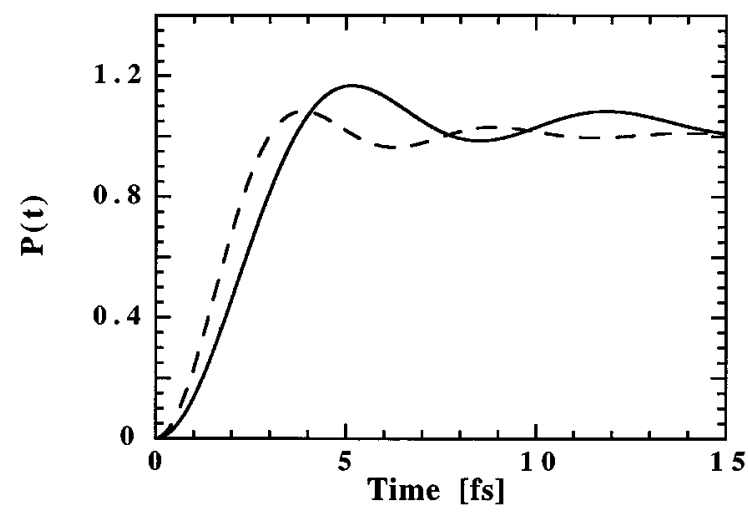

FIG. 9. Intervalley optical phonon: scattering rates for $\omega_{k}=\omega_{0}=4.7 \times 10^{13} \mathrm{~s}^{-1}$ when $\gamma=0.6 \times 10^{14} \mathrm{~s}^{-1}$ (solid line) and $\gamma=1.5 \times 10^{14} \mathrm{~s}^{-1}$ (dashed line).

non potential, is created and then decays. Furthermore, the collision duration is shown to decrease with increasing the initial-state lifetime and the final-state energy. In the polaroptical case, the behavior of the collision duration time as a function of the final-state frequency $\omega_{k}$ is similar to the Fermi liquid $2 \pi / \omega_{k}$ trend in the high-energy region, but has a much slower divergence in the low-energy region. This suggests that the latter is not a meaningful approach in the lowdensity semiconductor regime.

\section{ACKNOWLEDGMENTS}

This work has been supported by the Office of Naval Research. The authors would like to thank E. Ihrig for helpful discussions. The authors are also indebted to the reviewer of the manuscript for many useful suggestions, not the least of which was calling their attention to Ref. 24, which greatly simplified the calculations.
*Present address: Dipartimento di Fisica ed Istituto Nazionale di Fisica della Materia, Universitá di Modena, Via Campi 213/A, 41100 Modena, Italy.

${ }^{1}$ D. Emin, Phys. Rev. Lett. 25, 1751 (1970).

${ }^{2}$ D. Emin and A. M. Kriman, Phys. Rev. B 34, 7278 (1986).

${ }^{3}$ T. Holstein, Ann. Phys. (N.Y.) 8, 325 (1959).

${ }^{4}$ H. J. de Wit, Philips Res. Rep. 23, 449 (1968).

${ }^{5}$ S. Geltman, J. Phys. B 10, 831 (1977).

${ }^{6}$ S. L. Haan and S. Geltman, J. Phys. B 15, 1229 (1982).

${ }^{7}$ A. M. Lane, Phys. Lett. 99A, 359 (1983).

${ }^{8}$ M. H. Mittleman and A. Tip, J. Phys. B 17, 571 (1984).

${ }^{9}$ E. J. Robinson, Phys. Rev. Lett. 52, 2309 (1984).

${ }^{10}$ P. Lipavsky, F. S. Khan, A. Kalvová, and J. W. Wilkins, Phys. Rev. B 43, 6650 (1991).

${ }^{11}$ See, e.g., J. Shah, Solid State Electron. 32, 1051 (1989), and references contained therein.

${ }^{12}$ P. C. Becker, H. Fragnito, C. Brito-Cruz, J. Shah, R. L. Fork, J. E. Cunningham, J. E. Henry, and C. V. Shank, Phys. Rev. Lett. 61, 1647 (1988).

${ }^{13}$ A. M. Kriman, M. J. Kann, D. K. Ferry, and R. Joshi, Phys. Rev. Lett. 56, 1619 (1990).
${ }^{14}$ D. K. Ferry, A. M. Kriman, H. Hida, and S. Yamaguchi, Phys. Rev. Lett. 67, 633 (1991).

${ }^{15}$ T. Kuhn and F. Rossi, Phys. Rev. Lett. 69, 977 (1992); Phys. Rev. B 46, 7496 (1992).

${ }^{16} \mathrm{H}$. Haug, in Optical Nonlinearity and Instabilities in Semiconductors, edited by H. Haug (Academic, San Diego, 1988), p. 53.

${ }^{17}$ H. Haug, Phys. Status Solidi B 173, 139 (1992).

${ }^{18}$ G. D. Mahan, Many-Particle Physics (Plenum, New York, 1990).

${ }^{19}$ G. D. Mahan, Phys. Rep. 145, 251 (1987); J. Rammer and H. Smith, Rev. Mod. Phys. 58, 323 (1986).

${ }^{20}$ D. K. Ferry and H. L. Grubin, Solid State Physics, edited by H. Ehrenreich (Academic, New York, in press).

${ }^{21}$ P. Lipavsky, V. Spická, and B. Velicky, Phys. Rev. B 34, 6933 (1986).

${ }^{22}$ See, e.g., W. T. Thomson, Laplace Transformation (Prentice-Hall, Englewood Cliffs, NJ, 1960).

${ }^{23}$ R. R. Lewis, Phys. Rev. 102, 537 (1956).

${ }^{24}$ L. Bányai, D. B. Tran Thoai, C. Remling, and H. Haug, Phys. Status Solidi B 173, 149 (1992).

${ }^{25}$ C. Jacoboni and P. Lugli, The Monte Carlo Method for Semiconductor Device Simulation (Springer-Verlag, Wien, 1989). 